\title{
The spectral dimension of random trees
}

\author{
Claudio Destri and Luca Donetti \\ Dipartimento di Fisica G Occhialini, Università di Milano-Bicocca and INFN, Sezione di Milano, \\ Piazza delle Scienze 3, I-20126 Milano, Italy \\ E-mail: Claudio.Destri@mib.infn.it and Luca.Donetti@mib.infn.it
}

Received 17 June 2002, in final form 9 September 2002

Published 29 October 2002

Online at stacks.iop.org/JPhysA/35/9499

\begin{abstract}
We present a simple yet rigorous approach to the determination of the spectral dimension of random trees, based on the study of the massless limit of the Gaussian model on such trees. As a by-product, we obtain evidence in favour of a new scaling hypothesis for the Gaussian model on generic bounded graphs and in favour of a previously conjectured exact relation between spectral and connectivity dimensions on more general tree-like structures.
\end{abstract}

PACS number: $02.50 .-\mathrm{r}$

\section{Introduction and summary}

The spectral dimension $\bar{d}$ was first introduced by Alexander and Orbach [1] to characterize the low frequency vibration spectrum of fractals. Nowadays, it is considered by most the right generalization of the Euclidean dimension of regular lattices to irregular structures in general, whether fractal or not, such as polymers, glasses, percolation clusters, dendritic growths and so on. This is verified at least in many experimental situations, as well as in some theoretical model examples, whenever the physical dimensionality explicitly enters observables such as the spectrum of density fluctuations, the long-time properties of random walks, the critical exponents of statistical models and many others.

In a theoretical setting, we may consider a connected graph as a model of generic irregular structure, with physical microscopic degrees of freedom attached to the nodes and interaction among them associated with the links. This scheme implies that the coordination of nodes is bounded (the number of nearest neighbours of an atom, molecule or basic building block has a geometrical upper bound) and, if the graph in question is infinite, that the surface of any Van Hove ball (defined in terms of the chemical distance alone) must be negligible with respect to the volume when its radius goes to infinity. In other, more physically immediate words, we say that the structure is embeddable as a whole in a finite-dimensional Euclidean space and a standard thermodynamic limit of infinite systems exists. We are thus led to consider bounded graphs [2] and to study their spectral dimension. 
To be precise, we refer here to the average spectral dimension, that is a global property of the graph, related for instance to the infrared singularity of the Gaussian process or, equivalently, to the density of small eigenvalues of the Laplacian or the graph average of the long-time tail of the random walk return probability [3]. On macroscopically inhomogeneous structures this average dimension may differ from the local one, which enters the long-time tail of the random walk return probability on a given node [3]. However, it is the average spectral dimension that plays the same role of the lattice Euclidean dimension in many contexts, as for instance in providing a consistent criterion on whether a continuous symmetry breaks down at low temperatures [4].

In this work, we address the problem of determining the average spectral dimension of bounded trees and in particular of statistically homogeneous random trees [2] (this homogeneity actually implies that local and average spectral dimensions coincide), which are defined by the following branching algorithm: given a set of coordination fractions $\left\{f_{z}, 1 \leqslant z \leqslant \bar{z}\right\}$ that are properly normalized and have average coordination 2

$$
\sum_{z=1}^{\bar{z}} f_{z}=1 \quad \sum_{z=1}^{\bar{z}} z f_{z}=2
$$

an infinite random tree is built by first selecting with probability $f_{z}$ the coordination of the origin and then, proceeding shell by shell away from the origin, by choosing the coordination of every new node with probability $f_{z}$ except for the first node of each shell, when the modified probability $\tilde{f}_{z}=(z-1) f_{z}$ is used to implement the non-extinction preconditioning (this is algorithm $\mathbf{B}$ of [2]). These random trees represent a generalization of the incipient infinite percolation clusters on Bethe lattices [5], since the branching probabilities are freely assigned rather than being fixed by a single percolation probability. They also generalize to arbitrary coordination fractions $f_{z}$ the infinitely large specimens of the branched polymers studied for instance in [6].

Since the introduction of spectral dimension, the value of $\bar{d}=4 / 3$ has been conjectured for the incipient infinite percolation cluster in any dimension. This conjecture was proved false at low dimensions, while has been considered valid in the limit of infinite dimension (percolation on Bethe lattices). Leyvraz and Stanley [7] had in fact produced a heuristic scaling argument in favour of $\bar{d}=4 / 3$ already in 1983. Several years later, a more rigorous approach was pursued in [6], where the branched polymer phase of two-dimensional quantum gravity was proved to have indeed $\bar{d}=4 / 3$. Branched polymers correspond to a grand canonical ensemble of trees with all possible sizes, which is generated by a branching process like ours above but without the non-extinction preconditioning. In this case the definition of spectral dimension itself is subtle since such a concept does not apply to finite graphs. This is the major difficulty in the approach of [6]. Another mathematically well-founded approach to the problem is given in [8], where the exponent $\theta$ of the anomalous diffusion on our class of random trees is determined as $\theta=1$ (the author reports only the shorter and simpler of the two different derivations, which turns out to be more than 20 pages long, and states that the other, more complete treatment is 'monstrously long'). It is commonly held, although never rigorously established in general, that $\theta$ is connected to the spectral dimension and the (average) connectivity (or intrinsic Hausdorff) dimension $\bar{d}_{\mathrm{c}}$ as

$$
\frac{1}{2+\theta}=\frac{\bar{d}}{2 \bar{d}_{\mathrm{c}}}
$$

Therefore $\theta=1$ would imply $\bar{d}=4 / 3$, since $\bar{d}_{\mathrm{c}}=2$ for random trees [2].

In this paper, we provide a new, independent and fairly rigorous derivation of $\bar{d}=4 / 3$ based on the infrared properties of the Gaussian model on our random trees (section 5). 
The connection of the Gaussian model with the random walk on any graph is a well-known fact that we briefly review for completeness in section 2 . As a by-product, we also obtain an argument in favour of the conjecture (2), for any infinite graph for which the thermodynamic limit of the Gaussian model exists: it is natural in fact to expect the scaling form

$$
\mu \sim \mu_{0} r^{\bar{d}_{\mathrm{c}}} f\left(\mu_{0} r^{\gamma}\right)
$$

for the effective squared mass of the Gaussian model in terms of a very small 'bare' squared mass $\mu_{0}$ and the very large radius of a graph (see section 2 for the proper definitions); moreover we also conjecture that the finite-size exponent $\gamma$ coincides with $2+\theta$, which is sometimes called the random walk dimension and characterizes the scaling $t \sim r^{2+\theta}$ between the time needed by a random walker to visit with non-negligible frequency nodes at distance $r$ from its starting point; then the existence of the thermodynamic limit on the effective mass implies that $f(x) \sim x^{-\bar{d}_{\mathrm{c}} / \gamma}$ and so

$$
\mu \sim \mu_{0}^{1-\bar{d}_{\mathrm{c}} / \gamma}
$$

yielding exactly equation (2), since in general $\mu \sim \mu_{0}^{1-\bar{d} / 2}$ as $\mu_{0} \rightarrow 0$ (see section 2 ).

In our treatment of random trees we are indeed able to explicitly derive the scaling law (3) with $\bar{d}_{\mathrm{c}}=2$ and $\gamma=\bar{d}_{\mathrm{c}}+1=3$, demonstrating $\bar{d}=4 / 3$. It is interesting to note that if the scaling law (3) is assumed a priori, with $\bar{d}_{\mathrm{c}}=2$ as well known for random trees, then the precise numerical value of $\gamma$ (and therefore of $\bar{d}$ ) follows from a very simple 'geometrical' fact: as shown in section 5, the first correction to the finite tree behaviour $\mu \propto \mu_{0} r^{\bar{d}_{\mathrm{c}}}$ comes from the sum of squared volumes of all subtrees, which grows like $r^{2 \bar{d}_{\mathrm{c}}+1}$ if the volume of the tree grows like $r^{\bar{d}_{\mathrm{c}}}$. Then

$$
\bar{d}=\frac{2 \bar{d}_{\mathrm{c}}}{\bar{d}_{\mathrm{c}}+1}
$$

which is also a known conjecture, even with $\bar{d}_{\mathrm{c}} \neq 2$, for the incipient percolation cluster and other tree-like disordered structures [9]. Note that $\bar{d}$ is monotonically increasing from 1 to $4 / 3$ as $\bar{d}_{\mathrm{c}}$ grows from 1 to 2 .

Let us observe that $\bar{d}_{\mathrm{c}}=2$ and $\bar{d}=4 / 3$ are the greatest values among all known average connectivity and spectral dimensions for bounded trees. This fact leads us to formulate the following conjecture: for all bounded trees $\bar{d}_{\mathrm{c}} \leqslant 2$ and $\bar{d} \leqslant \frac{4}{3}$, with saturation only in the case of random trees. Evidently this must be true only for the average dimensions; there indeed exist examples of trees (such as $N T_{D}$ [10]) whose local connectivity and spectral dimension, $d_{\mathrm{c}}$ and $d$, exceed 2 and 4/3, respectively; these present however macroscopic inhomogeneities so that the local dimensions are different from the average ones which do satisfy the bounds at 2 and 4/3. Let us also observe that equation (4) does not apply in the same examples if the average dimensions are replaced by the local ones.

At the moment these upper bounds are only conjectured, but it has been rigorously proved that $\bar{d} \leqslant 2$, since bounded trees are always recurrent on the average [11].

\section{Gaussian model, random walks and spectral dimension}

The Gaussian model on a generic connected infinite graph $\mathcal{G}$ is defined [12] by assigning a real-valued random variable $\phi_{x}$ to each node $x \in \mathcal{G}$ with the following probability distribution:

$$
\mathrm{d} v_{r}[\phi]=\frac{1}{Z_{r}} \exp \left[-\frac{1}{2} \sum_{x, y \in B(o, r)} \phi_{x}\left(L_{x y}+\mu_{0} \delta_{x y}\right) \phi_{y}\right] \prod_{x \in B(x, r)} \mathrm{d} \phi_{x}
$$


for the collection $\phi=\left\{\phi_{x} ; x \in B(o, r)\right\}$ relative to any Van Hove ball

$$
B(o, r)=\{y \in \mathcal{G}: d(o, y) \leqslant r\} \quad o \in \mathcal{G} \quad 0 \leqslant r<\infty
$$

where $d(o, y)$ is the chemical distance. In equation (5) $Z_{r}$ is the proper normalization factor (the partition function), $\mu_{0}>0$ is a free parameter (the squared mass in field-theoretic sense) and

$$
L_{x y}=z_{x} \delta_{x y}-A_{x y}
$$

is (minus) the Laplacian matrix on $\mathcal{G}, z_{x}$ being the coordination or degree of $x$ and $A_{x y}$ the adjacency matrix).

The thermodynamic limit is achieved by letting $r \rightarrow \infty$ and defines a Gaussian measure for the whole field $\phi=\left\{\phi_{x} ; x \in \mathcal{G}\right\}$ which does not depend on the centre $o$ of the ball [13] if $\mathcal{G}$ is bounded. The covariance of this Gaussian process reads

$$
\left\langle\phi_{x} \phi_{y}\right\rangle \equiv C_{x y}\left(\mu_{0}\right)=\left(\boldsymbol{L}+\mu_{0}\right)_{x y}^{-1}
$$

and setting

$$
C_{x y}=\frac{(1-\boldsymbol{W})_{x y}^{-1}}{z_{x}+\mu_{0}} \quad W_{x y}=\frac{A_{x y}}{z_{y}+\mu_{0}}
$$

one obtains the standard connection with the random walks over $\mathcal{G}$ [12]:

$$
(1-\boldsymbol{W})_{x y}^{-1}=\sum_{t=0}^{\infty}\left(\boldsymbol{W}^{t}\right)_{x y}=\sum_{\gamma: x \leftarrow y} W[\gamma]
$$

where the last sum runs over all paths from $y$ to $x$, each weighted by the product along the path of the one-step jump probabilities in $\boldsymbol{W}$ :

$\gamma=\left(x, y_{t-1}, \ldots, y_{2}, y_{1}, y\right) \quad \Longrightarrow \quad W[\gamma]=W_{x y_{t-1}} W_{y_{t-1} y_{t-2}}, \ldots, W_{y_{2} y_{1}} W_{y_{1} y}$.

Note that, as long as $\mu_{0}>0$, we have $\sum_{x}\left(\boldsymbol{W}^{t}\right)_{x y}<1$ for any $t$, namely the walker has a nonzero death probability. This implies that $C_{x y}$ is a smooth function of $\mu_{0}$ for $\mu_{0} \geqslant \epsilon>0$. In the limit $\mu_{0} \rightarrow 0$ the walker never dies and the standard random walk is recovered; then the sum over paths in equation (9) is dominated by the infinitely long paths which sample the large scale structure of the entire graph ('large scale' refers here to the metric induced by the chemical distance alone). This typically reflects itself into a singularity of $C_{x y}$ at $\mu_{0}=0$. Consider for instance a diagonal element $C_{x x}$ of the covariance and suppose that its singularity is power-like

$$
\text { Sing } C_{x x}\left(\mu_{0}\right) \propto \mu_{0}^{d / 2-1} .
$$

Then by equation (8) one sees that the same power-like behaviour appears, as $\lambda \rightarrow 1$, in the discrete Laplace transform $P_{x x}(\lambda)$ of the random walks return probability $P_{x x}(t)$ at $x$. In turn this implies the long-time behaviour

$$
P_{x x}(t) \propto t^{-d / 2} \quad t \rightarrow \infty
$$

identifying $d$ with the local spectral dimension, which does not depend on the specific node $x$ [12]. To be precise the denomination 'spectral dimension' refers to the behaviour of the spectral density $\rho(l)$ of low-lying eigenvalues of the Laplacian $\boldsymbol{L}$, namely $\rho(l) \sim l^{\bar{d} / 2-1}$. In fact, it can be shown [3] that $\bar{d}$ is connected to the long-time behaviour of the graph average of $P_{x x}(t)$, or equivalently to the infrared power-like singularity of the graph average $\overline{C\left(\mu_{0}\right)}$ of $C_{x x}$, that is

$$
\operatorname{Sing} \overline{C\left(\mu_{0}\right)}=\text { Sing } \lim _{r \rightarrow \infty} \frac{1}{|B(o, r)|} \sum_{x \in B(o, r)} C_{x x}\left(\mu_{0}\right) \propto\left(\mu_{0}\right)^{\bar{d} / 2-1}
$$


where $|B(o, r)|$ is the volume of $B(o, r) . \bar{d}$ is therefore sometimes called the 'average' spectral dimension. On regular lattices it coincides with the local spectral dimension and the usual Euclidean dimension.

By definition, the diagonal elements $C_{x x}$ of the covariance may be written as

$$
C_{x x}=\int \mathrm{d} \varphi \varphi^{2} \mathcal{Z}_{x}(\varphi)
$$

where

$$
\mathcal{Z}_{x}(\varphi)=\lim _{r \rightarrow \infty} \int \mathrm{d} v_{r}[\phi] \delta\left(\phi_{x}-\varphi\right)
$$

Thanks to the fundamental self-reproducing property of Gaussian integrations, $\mathcal{Z}_{x}(\varphi)$ is necessarily a normalized Gaussian in $\varphi$, that is

$$
\mathcal{Z}_{x}(\varphi)=\left(\frac{\mu_{x}}{2 \pi}\right)^{1 / 2} \mathrm{e}^{-\mu_{x} \varphi^{2} / 2}
$$

where, for consistency with equation (14)

$$
\mu_{x}\left(\mu_{0}\right)=\frac{1}{2 C_{x x}\left(\mu_{0}\right)}
$$

is the 'effective mass' at $x$.

\section{The Gaussian model on random trees}

Let us now consider the Gaussian model on an infinite bounded tree $\mathcal{T}$. Any node $x \in \mathcal{T}$ may be regarded as the root of an infinite branching process which produces $\mathcal{T}$ as a family tree rooted at $x$. This simply means that $x$ has as many descendants, or children, as its coordination $z_{x}$, while any other node $x^{\prime}$ has $z_{x^{\prime}}-1$ children one step further away from the unique ancestor $x$ and a father one step closer to $x$. In particular, the $z_{x}$ children $y$ of $x$ are themselves roots of disjoint subtrees, or branches $\mathcal{T}_{y}^{\prime}$, which may or may not be infinite. Now, by the simple rules of Gaussian integration, one easily verifies the following relation between the effective mass at $x$, relative to the entire $\mathcal{T}$ and the effective masses at children $y$ of $x$, each one relative to the corresponding branch $\mathcal{T}_{y}^{\prime}$ :

$$
\mu_{x}[\mathcal{T}]=\mu_{0}+\sum_{\substack{y \text { children } \\ \text { of } x}} \frac{\mu_{y}\left[\mathcal{T}_{y}^{\prime}\right]}{1+\mu_{y}\left[\mathcal{T}_{y}^{\prime}\right]}
$$

A similar relation holds for the effective masses $\mu_{y}\left[\mathcal{T}_{y}^{\prime}\right]$ in terms of the $z_{y}-1$ masses of $y$ 's children, and so on for the rest of the tree.

Next, suppose that the branching process is the Galton-Watson random one, with the non-extinction preconditioning, described in the introduction (and in more detail in [2]), so that $\mathcal{T}$ is an infinite random tree. Because of the preconditioning, $\mathcal{T}$ may be regarded as the union of infinitely many subtrees generated by independent and identically distributed (i.i.d.) random branching processes, without the non-extinction preconditioning, whose roots are attached to the nodes of a half-infinite chain (the spine) that starts from $x$. This means that only one of the branches at $x$ is a priori guaranteed to extend to infinity, while the other $z_{x}-1$ are the family trees of i.i.d. random branching processes which might stop after finitely many generations.

Let us consider first the probability distributions relative to the branching process itself and in particular the probability $P_{r}(\mu)$ that the effective mass at the root $x$ be exactly $\mu$ when the branching algorithm is iterated for $r$ generations, that is

$$
P_{r}(\mu)=\left\langle\delta\left(\mu_{x}-\mu\right)\right\rangle_{r}
$$


where the average is over all realizations of the tree with $r$ generations, namely on all possible histories of the branching algorithm with $r$ shells completed. Taking equation (16) and the spinal decomposition into account, we may write

$P_{r+1}(\mu)=\sum_{z} f_{z} \int \mathrm{d} \mu_{1} \mathrm{~d} \mu_{2} \cdots \mathrm{d} \mu_{z} \delta\left(\mu-\mu_{0}-\sum_{j=1}^{z} \frac{\mu_{j}}{1+\mu_{j}}\right) P_{r}^{\bullet}\left(\mu_{1}\right) \prod_{j=2}^{z} P_{r}^{\circ}\left(\mu_{j}\right)$

where $P^{\circ}(\mu)$ is the same probability without the non-extinction preconditioning and fulfils the functional recursion

$P_{r+1}^{\circ}(\mu)=\sum_{z} f_{z} \int \mathrm{d} \mu_{1} \mathrm{~d} \mu_{2} \cdots \mathrm{d} \mu_{z-1} \delta\left(\mu-\mu_{0}-\sum_{j=1}^{z-1} \frac{\mu_{j}}{1+\mu_{j}}\right) \prod_{j=1}^{z-1} P_{r}^{\circ}\left(\mu_{j}\right)$

while $P_{r}^{\bullet}(\mu)$ is the probability when also the coordination of the root is extracted with the modified probability $\tilde{f}_{z}$ and satisfies therefore

$P_{r+1}^{\bullet}(\mu)=\sum_{z} \tilde{f}_{z} \int \mathrm{d} \mu_{1} \mathrm{~d} \mu_{2} \cdots \mathrm{d} \mu_{z-1} \delta\left(\mu-\mu_{0}-\sum_{j=1}^{z-1} \frac{\mu_{j}}{1+\mu_{j}}\right) P_{r}^{\bullet}\left(\mu_{1}\right) \prod_{j=2}^{z-1} P_{r}^{\circ}\left(\mu_{j}\right)$.

As $r \rightarrow \infty$ we expect all these probability distributions to converge to their limiting forms $P_{\infty}(\mu), P_{\infty}^{\circ}(\mu)$ and $P_{\infty}^{\bullet}(\mu)$, turning equations (17), (18) and (19) into highly nontrivial coupled integro-functional equations for $P_{\infty}(\mu), P_{\infty}^{\circ}(\mu)$ and $P_{\infty}^{\bullet}(\mu)$ with a parametric dependence on $\mu_{0}$. In particular, we are interested in their infrared behaviour as $\mu_{0} \rightarrow 0$.

\section{Statistical homogeneity and auto-averaging property}

Before addressing the problem just mentioned, we need to establish the precise relation with the original problem, that of determining the $\mu_{0} \rightarrow 0$ behaviour of $\overline{C\left(\mu_{0}\right)}$ on a given 'generic' infinite random tree $\mathcal{T}$ produced by our algorithm. By 'generic' in this context we mean an infinite $\mathcal{T}$ belonging to the subset of unit measure of trees that fulfil the auto-averaging property for local observables. To be explicit, consider the frequency $F(r, \tau)$ of occurrence in $\mathcal{T}$ of a given rooted tree $\tau$ with $r$ generations. In terms of the Van Hove balls $B(x, r)$, regarded as subtrees of $\mathcal{T}$, we may write $F(r, \tau)$ as the graph average

$$
F(r, \tau)=\lim _{R \rightarrow \infty} F_{R}(r, \tau) \quad F_{R}(r, \tau)=\frac{1}{|B(o, R)|} \sum_{x \in B(o, R)} \delta[B(x, r)=\tau]
$$

where $o$ is an arbitrary node of $\mathcal{T}$ and $\delta\left[\tau^{\prime}=\tau\right]$ is one if its argument is a true statement and zero otherwise. $F(r, \tau)$ does not depend on the choice of $o(\mathcal{T}$ is a bounded graph) and it is convenient to identify $o$ as the root, or origin, of the infinite branching algorithm.

Let us then recall that any $\mathcal{T}$ produced by the algorithm may be decomposed into the spine $\mathcal{S}$ (the origin $o$ plus all the nodes on which the coordination was extracted with $\tilde{f}_{z}$ ) and the transverse family trees of the identical, independent and non-preconditioned branching processes starting from the nodes of the spine (see [2, 8] for more details). Evidently, the intersection of the transverse family trees rooted at $s \in \mathcal{S}$ with $B(o, R)$ contains at most $R-d(s, o)$ generations, so that they contribute differently, even on average, to $F_{R}(r, \tau)$. Therefore, to simplify our derivation, we consider a modified thermodynamic limit, in which the infinite tree is not recovered through a Van Hove ball with diverging radius, but as $R \rightarrow \infty$ limit of the set

$$
\Omega_{R}=\bigcup_{s \in \mathcal{S}_{R}} \omega_{s, R}
$$


where $S_{R}$ is the restriction of the spine to the first $R$ generations of $\mathcal{T}$ and $\omega_{s, R}$ is the union of all transverse family trees rooted at $s$ and restricted to the first $R$ generations. Thus we redefine $F_{R}(r, \tau)$ as

$$
\begin{aligned}
& F_{R}(r, \tau)=\sum_{s \in \mathcal{S}_{R}} w_{s, R} Q_{s, R}(r, \tau) \quad w_{s, R}=\frac{\left|\omega_{s, R}\right|}{\left|\Omega_{R}\right|} \\
& Q_{s, R}(r, \tau)=\frac{1}{\left|\omega_{s, R}\right|} \sum_{x \in \omega_{s, R}} \delta[B(x, r)=\tau] .
\end{aligned}
$$

Note that, by construction, the random weights $w_{s, R}$ are all identically distributed and are all independent except for the normalization constraint $\sum_{s \in \mathcal{S}_{R}} w_{s, R}=1$. The random frequencies $Q_{s, R}(r, \tau)$ are all identically distributed, but are not independent because a ball $B(x, r)$ will in general intersect several $\omega_{s, R}$ if $d(x, s)<r$. Therefore we split $\omega_{s, R}$ into $\omega_{s, R}^{>}$, formed by nodes $x$ such that $d(x, s)>r$, and its complement $\omega_{s}^{\leqslant}$, which does not depend on $R$ for large $R$. This implies an analogous splitting for the random weights and the random frequencies. Now the random frequencies $Q_{s, R}^{>}(r, \tau)$ are all independent since the two events $B(x, r)=\tau$ and $B\left(x^{\prime}, r\right)=\tau$ are independent if $x \in \Omega_{s, R}^{>}$and $x^{\prime} \in \Omega_{s^{\prime}, R}^{>}$with $s \neq s^{\prime}$. Note also that $Q_{s, R}(r, \tau)$ and $w_{s^{\prime}, R}$ are independent when $s \neq s^{\prime}$.

Thus we have

$$
F_{R}(r, \tau)=F_{R}^{\leqslant}(r, \tau)+F_{R}^{>}(r, \tau)
$$

with $F_{R}^{\leqslant}(r, \tau)$ getting contributions solely from nodes at distances not larger than $r$ from the spine. Clearly, in the limit $R \rightarrow \infty$, these nodes form a subset of zero measure of $\mathcal{T}$, since $\bigcup_{s} \omega_{s}^{\leqslant}(r, \tau)$ is essentially one dimensional while a generic $\mathcal{T}$ has $\bar{d}_{\mathrm{c}}=2$. Hence, as $R \rightarrow \infty$,

$$
F(r, \tau)=\lim _{R \rightarrow \infty} F_{R}^{>}(r, \tau)
$$

By analogous dimensionality arguments we get that almost always $\lim _{R \rightarrow \infty} w_{s, R}=0$, although the sum of all these random weights stays properly normalized.

We may now invoke directly the law of large numbers for $F_{R}^{>}(r, \tau)$, which is the weighted average of i.i.d. random variables. Hence, for any finite $r, F(r, \tau)$ is almost always nonfluctuating on infinite trees and

$$
F(r, \tau)=\left\langle Q^{>}(r, \tau)\right\rangle^{\circ}
$$

where $Q^{>}(r, \tau)$ is any one of the $\lim _{R \rightarrow \infty} Q_{s, R}^{>}(r, \tau)$ and the average $\langle\cdot\rangle^{\circ}$ is taken with respect to the algorithm without the preconditioning on non-extinction. But any node $x$ on such trees lying at a distance larger then $r$ from the origin enjoys statistical homogeneity for the event $B(x, r)=\tau$, since the branching is i.i.d. on every node (but the origin, at most) and $B(x, r)=\tau$ cannot contain the origin. Moreover, the unique path connecting $x$ to $o$ plays the role of the spine for a branching process rooted at $x$ and preconditioned on non-extinction for at least $r$ generations, since the trees contributing to $\left\langle Q^{>}(r, \tau)\right\rangle^{\circ}$ have at least $r$ generations. We conclude therefore that almost always an infinite random tree $\mathcal{T}$ has the auto-averaging property

$$
F(r, \tau)=\langle\delta[B(o, r)=\tau]\rangle
$$

with respect to the probability itself that the first $r$ shells of $\mathcal{T}$ build up to form the given finite tree $\tau$. Then this property holds for any local observable and in particular for the effective mass $\mu_{x}[B(x, r)]$ of a Gaussian model restricted to $B(x, r)$. Thus the probability distribution $P_{r}(\mu)$ coincides with the frequency of the event $\mu_{x}[B(x, r)]=\mu$, as $x$ varies throughout a 'generic' infinite random tree $\mathcal{T}$. The same would evidently apply to the probability distribution of the variable $(2 \mu)^{-1}$ and the frequency of the event $C_{x x}[B(x, r)]=(2 \mu)^{-1}$. 


\section{Scaling}

Let us now return to the recursion relations (17), (18) and (19) for the probability distributions $P_{r}(\mu), P_{r}^{\circ}(\mu)$ and $P_{r}^{\bullet}(\mu)$, respectively. We are interested in the thermodynamic limit $r \rightarrow \infty$ of infinite trees followed by the massless limit $\mu_{0} \rightarrow 0$. These two limits can actually be applied simultaneously, provided we identify the correct scaling variables. Suppose in fact that, as $r \rightarrow \infty$ and $\mu, \mu_{0} \rightarrow 0$, the scaling law (3) applies; since $\mu$ is really a random variable, a better formulation is in terms of its probability, that is

$$
P_{r}\left(\mu ; \mu_{0}\right) \simeq \mu_{0}^{\beta} F\left(\mu \mu_{0}^{\beta}, \mu_{0} r^{\gamma}\right)
$$

for suitable scaling exponents $\beta$ and $\gamma$ and scaling function $F(u, s) . \beta$ is then directly related to the spectral dimension, as $\beta=-1+\bar{d} / 2$, thanks to equation (11), the results of the previous section and the existence of the thermodynamic limit; $\gamma$ provides instead a measure of finitesize effects at very large sizes. In particular, if we let $\mu_{0} \rightarrow 0$ too fast, so that $\mu_{0} r^{\gamma} \rightarrow 0$ even if $r \rightarrow \infty$, we must recover the massless behaviour characteristic of finite trees. This amounts to linearizing the effective mass formula (16), so that

$$
\mu_{x}[\mathcal{T}]=\mu_{0} v[\mathcal{T}]
$$

where $v[\mathcal{T}]$ is the volume of $\mathcal{T}$. Thus

$$
P_{r}\left(\mu ; \mu_{0}\right) \simeq \mu_{0}^{-1} \hat{P}_{r}\left(\mu / \mu_{0}\right)=\mu_{0}^{-1} \hat{P}_{r}(v)
$$

where $\hat{P}_{r}(v)$ is the volume probability at radius $r$, which for large $r$ has a scaling form in terms of $v / r^{2}$ explicitly determined in [2] (thus implying $\bar{d}_{\mathrm{c}}=2$ ). Since this scaling form is highly nontrivial, with exponentially small behaviour also for $v / r^{2} \rightarrow 0$, compatibility with the scaling hypothesis (21) requires $1+\beta=2 / \gamma$, that is

$$
\frac{1}{\gamma}=\frac{\bar{d}}{4}=\frac{\bar{d}}{2 \bar{d}_{\mathrm{c}}} .
$$

One is therefore led to the identification (compare with equation (2) in the introduction)

$$
\gamma=2+\theta
$$

relating finite-size effects to the exponent of anomalous diffusion. Of course, since relation (2) is only a well-founded conjecture, an explicit derivation of $\gamma=2+\theta$ would provide a proof of the conjecture for our random trees. Let us stress the point: $\gamma$ measures the scaling behaviour of the Gaussian model (and therefore of the random walk) on finite random trees of very large radius $r ; 2+\theta$ characterizes instead the scaling $t \sim r^{2+\theta}$ between the time needed by a random walker to visit with non-negligible frequency nodes at distance $r$ from its starting point. The two concepts are certainly related but quite distinct, so that the identification $\gamma=2+\theta$ has a nontrivial content.

Let us come back now to the opposite limiting behaviour of the probability distributions that is the subject of this work, namely $r \rightarrow \infty$ and $\mu, \mu_{0} \rightarrow 0$ with $u=\mu \mu_{0}^{\beta}$ fixed and $s=\mu_{0} r^{\gamma}$ finite and possibly very large. We shall show now that the scaling law (21) is indeed correct with the precise values

$$
\beta=-1 / 3 \quad \gamma=3
$$

implying $\bar{d}=4 / 3$. The detailed proof, by induction on all moments of $P_{r}(\mu), P_{r}^{\circ}(\mu)$ and $P_{r}^{\bullet}(\mu)$, is rather simple albeit notationally a bit cumbersome and is reported in the appendix. Here we only provide a sketch of the derivation.

The first step consists in rewriting the recursion relations (17), (18) and (19) into a more tractable form. In fact they are amenable to a direct treatment via Laplace transform only in 
the limit $\mu_{0} \rightarrow 0$ at fixed $r$, while we are interested in a regime where the nonlinearity in the effective mass formula (16) plays a crucial role. Therefore we consider the expansion in all powers of $\mu_{0}$

$$
\mu_{x}[\mathcal{T}]=\sum_{n=1}^{\infty}(-1)^{n+1} V_{n}(x) \mu_{0}^{n}
$$

where the coefficients $V_{n}(x)$ are integer-valued random variables satisfying composition laws obtained by equating powers of $\mu_{0}$ on both sides of equation (16). These read

$$
\begin{aligned}
& V_{1}(x)=1+\sum_{\substack{y \text { children } \\
\text { of } x}} V_{1}(y) \\
& V_{2}(x)=\sum_{\substack{y \text { children } \\
\text { of } x}}\left[V_{2}(y)+V_{1}(y)^{2}\right] \\
& V_{3}(x)=\sum_{\substack{y \text { children } \\
\text { of } x}}\left[V_{3}(y)+2 V_{1}(y) V_{2}(y)+V_{1}(y)^{3}\right] \\
& V_{4}(x)=\sum_{\substack{y \text { children } \\
\text { of } x}}\left[V_{4}(y)+2 V_{1}(y) V_{3}(y)+V_{2}(y)^{2}+3 V_{1}(y)^{2} V_{2}(y)+V_{1}(y)^{4}\right] \\
& V_{5}(x)=\cdots
\end{aligned}
$$

which we write in general as

$$
V_{n}(x)=\delta_{n, 1}+\sum_{\substack{y \text { children } \\ \text { of } x}} \mathcal{F}_{n}\left(V_{1}(y), V_{2}(y), \ldots, V_{n}(y)\right)
$$

where the form of $\mathcal{F}_{n}$ can be easily induced from the previous formulae. Note that $V_{1}$ can be interpreted as the volume of the subtree, $V_{2}$ as the sum of the squares of all sub-volumes and so on. Now we may write recursion relations for the probability of the coefficients $V_{n}(x)$ themselves. For instance, in the branching algorithm without non-extinction preconditioning, we have

$$
\begin{aligned}
P_{r+1}^{\circ}\left(V_{1}, \ldots, V_{n}\right) & =\sum_{z} f_{z} \sum_{\substack{V_{1,1}, \ldots, V_{1, z-1} \\
V_{2,1}, \ldots, V_{2, z-1} \\
V_{n, 1}, \ldots, V_{n, z-1}}}^{z-1} P_{r}^{\circ}\left(V_{1, i}, \ldots, V_{n, i}\right) \\
& \times \prod_{k=1}^{n} \delta\left(V_{k}-\delta_{n, 1}-\sum_{j=1}^{z-1} \mathcal{F}_{k}\left(V_{1, j}, \ldots, V_{k, j}\right)\right)
\end{aligned}
$$

with similar expressions for probabilities $P_{r}\left(V_{1}, \ldots, V_{n}\right)$ and $P_{r}^{\bullet}\left(V_{1}, \ldots, V_{n}\right)$ corresponding to those for the effective mass $P_{r}(\mu)$ and $P_{r}^{\bullet}(\mu)$. For brevity we do not write them here; they can be found in the appendix.

The advantage of this new formulation lies in the fact that $\mathcal{F}_{n}\left(\left\{V_{1, i}, \ldots, V_{n, i}\right)\right.$ is by construction linear in $V_{n, i}$. This allows us to set up a systematic control of the large $r$ behaviour of the recursion relations for the multiple moments of $P_{r}\left(V_{1}, \ldots, V_{n}\right), P_{r}^{\circ}\left(V_{1}, \ldots, V_{n}\right)$ and $P_{r}^{\bullet}\left(V_{1}, \ldots, V_{n}\right)$. Consider for example the lowest moments: from equation (25) and its analogues in the appendix we get 


$$
\begin{aligned}
& \left\langle V_{1}\right\rangle_{r+1}^{\circ}=\sum_{z} f_{z}\left(1+(z-1)\left\langle V_{1}\right\rangle_{r}^{\circ}\right)=1+\left\langle V_{1}\right\rangle_{r}^{\circ} \\
& \left\langle V_{1}\right\rangle_{r+1}^{\bullet}=\sum_{z}(z-1) f_{z}\left(1+\left\langle V_{1}\right\rangle_{r}^{\bullet}+(z-2)\left\langle V_{1}\right\rangle_{r}^{\circ}\right)=1+\left\langle V_{1}\right\rangle_{r}^{\bullet}+g^{\prime \prime}(1)\left\langle V_{1}\right\rangle_{r}^{\circ} \\
& \left\langle V_{1}\right\rangle_{r+1}=\sum_{z} f_{z}\left(1+\left\langle V_{1}\right\rangle_{r}^{\bullet}+(z-1)\left\langle V_{1}\right\rangle_{r}^{\circ}\right)=1+\left\langle V_{1}\right\rangle_{r}^{\bullet}+\left\langle V_{1}\right\rangle_{r}^{\circ}
\end{aligned}
$$

where $g(\lambda)$ is the so-called probability generating function

$$
g(\lambda)=\sum_{z} f_{z} \lambda^{z-1}=f_{1}+f_{2} \lambda+f_{3} \lambda^{2}+\cdots
$$

which thanks to equations (1) enjoys the properties

$$
g(1)=1 \quad g^{\prime}(1)=1 .
$$

Hence we obtain the asymptotic behaviour for large $r$

$$
\left\langle V_{1}\right\rangle_{r}^{\circ} \sim r \quad\left\langle V_{1}\right\rangle_{r} \sim\left\langle V_{1}\right\rangle_{r}^{\bullet} \sim r^{2}
$$

which implies $\bar{d}_{\mathrm{c}}=2$ since $V_{1}$ is just the volume. Next consider the averages of $V_{2}$ and $V_{1}^{2}$; the recursion rules read

$$
\begin{aligned}
\left\langle V_{2}\right\rangle_{r+1}^{\circ} & =\sum_{z}(z-1) f_{z}\left(\left\langle V_{2}\right\rangle_{r}^{\circ}+\left\langle V_{1}^{2}\right\rangle_{r}^{\circ}\right)=\left\langle V_{2}\right\rangle_{r}^{\circ}+\left\langle V_{1}^{2}\right\rangle_{r}^{\circ} \\
\left\langle V_{2}\right\rangle_{r+1}^{\circ} & =\sum_{z}(z-1) f_{z}\left[\left\langle V_{2}\right\rangle_{r}^{\bullet}+\left\langle V_{1}^{2}\right\rangle_{r}^{\bullet}+(z-2)\left\langle V_{2}\right\rangle_{r}^{\circ}+(z-2)\left\langle V_{1}^{2}\right\rangle_{r}^{\circ}\right] \\
& =\left\langle V_{2}\right\rangle_{r}^{\bullet}+\left\langle V_{1}^{2}\right\rangle_{r}^{\bullet}+g^{\prime \prime}(1)\left\langle V_{2}\right\rangle_{r}^{\circ}+g^{\prime \prime}(1)\left\langle V_{1}^{2}\right\rangle_{r}^{\circ} \\
\left\langle V_{2}\right\rangle_{r+1} & =\left\langle V_{2}\right\rangle_{r}^{\bullet}+\left\langle V_{1}^{2}\right\rangle_{r}^{\bullet}+\left\langle V_{2}\right\rangle_{r}^{\circ}+\left\langle V_{1}^{2}\right\rangle_{r}^{\circ}
\end{aligned}
$$

and

$\left\langle V_{1}^{2}\right\rangle_{r+1}^{\circ}=1+\left\langle V_{1}^{2}\right\rangle_{r}^{\circ}+g^{\prime \prime}(1)\left(\left\langle V_{1}\right\rangle_{r}^{\circ}\right)^{2}+2\left\langle V_{1}\right\rangle_{r}^{\circ}$

$\left\langle V_{1}^{2}\right\rangle_{r+1}^{\bullet}=1+\left\langle V_{1}^{2}\right\rangle_{r}^{\bullet}+2\left\langle V_{1}\right\rangle_{r}^{\bullet}+g^{\prime \prime}(1)\left[\left\langle V_{1}^{2}\right\rangle_{r}^{\circ}+\left\langle V_{1}\right\rangle_{r}^{\circ}\left\langle V_{1}\right\rangle_{r}^{\bullet}+2\left\langle V_{1}\right\rangle_{r}^{\circ}\right]+g^{\prime \prime \prime}(1)\left(\left\langle V_{1}\right\rangle_{r}^{\circ}\right)^{2}$

$\left\langle V_{1}^{2}\right\rangle_{r+1}=1+\left\langle V_{1}^{2}\right\rangle_{r}^{\bullet}+2\left\langle V_{1}\right\rangle_{r}^{\bullet}+\left\langle V_{1}^{2}\right\rangle_{r}^{\circ}+\left\langle V_{1}\right\rangle_{r}^{\circ}\left\langle V_{1}\right\rangle_{r}^{\bullet}+2\left\langle V_{1}\right\rangle_{r}^{\circ}+g^{\prime \prime}(1)\left(\left\langle V_{1}\right\rangle_{r}^{\circ}\right)^{2}$.

We can read right away the $r \rightarrow \infty$ asymptotic behaviour

$$
\begin{aligned}
\left\langle V_{1}^{2}\right\rangle_{r}^{\circ} & \sim r^{3} & \left\langle V_{2}\right\rangle_{r}^{\circ} & \sim r^{4} \\
\left\langle V_{1}^{2}\right\rangle_{r} & \sim r^{4} & \left\langle V_{2}\right\rangle_{r} & \sim r^{5} .
\end{aligned}
$$

Evidently the moments of $P_{r}^{\bullet}\left(V_{1}, \ldots, V_{n}\right)$ all have the same asymptotic behaviour as those of $P_{r}\left(V_{1}, \ldots, V_{n}\right)$ and need not be written out explicitly.

Let us make use of the results (28) and (29) for the mean value of the effective mass at the root $o$ :

$$
\langle\mu\rangle_{r}=\sum_{n=1}^{\infty}(-1)^{n+1}\left\langle V_{n}\right\rangle_{r} \mu_{0}^{n} \simeq \mu_{0} r^{2}\left(c_{0}+c_{1} \mu_{0} r^{3}+\cdots\right)
$$

where $c_{0}$ and $c_{1}$ are constants. If we assume the scaling law (21) for the probability $P_{r}\left(\mu ; \mu_{0}\right)$, then clearly

$$
\langle\mu\rangle_{r} \simeq \mu_{o}^{-\beta} F_{1}\left(\mu_{0} r^{\gamma}\right)
$$


with $F_{1}(s)$ finite in the limit $s \rightarrow \infty$; consistency with (31) now requires that $F_{1}(s) \sim$ $s^{2 / \gamma}\left(1+O\left(s^{3}\right)\right)$ as $s \rightarrow 0$ to reproduce the correct $r$ behaviour on large but finite trees; but matching also the powers of $\mu_{0}$ implies

$$
1+\beta=2 / \gamma \quad 2+\beta=5 / \gamma
$$

yielding exactly the desired result (22). This argument may be turned around to avoid referring to the scaling hypothesis (21) for the probability and to better clarify the role of the thermodynamic limit. In fact, the first two terms of the $\mu_{0}$-expansion in (31) suggest that, as $r \rightarrow \infty$ and $\mu_{0} \rightarrow 0$ at fixed $\mu_{0} r^{3}$,

$$
\langle\mu\rangle_{r} \simeq \mu_{0} r^{2} G_{1}\left(\mu_{0} r^{3}\right)
$$

for a suitable scaling function $G_{1}(s)$. Now the existence of the thermodynamic limit [12] requires that $G_{1}(s) \sim s^{-2 / 3}$ for large $s$, yielding again $\langle\mu\rangle_{\infty} \sim \mu_{0}^{1 / 3}$, that is $\beta=-1 / 3$. Of course this formulation is only tentative, since we still need to verify that, for any other $n>2$, the $n$th term in the $\mu_{0}$-expansion of $\langle\mu\rangle_{r}$ contains $r^{3 n+2}$ as the highest power of $r$. Moreover, even if we can prove the scaling (32) of $\langle\mu\rangle_{r}$, this does not imply the scaling (21) for the whole probability $P_{r}\left(\mu ; \mu_{0}\right)$. In the appendix we demonstrate by induction that, as $r \rightarrow \infty$

$$
\left\langle\prod_{j=1}^{k} V_{n_{j}}\right\rangle_{r} \sim r^{3 \sum_{j} n_{j}-k}\left[1+O\left(r^{-1}\right)\right]
$$

Hence, by retaining only the dominant $r$ behaviour, we have

$$
\langle\mu\rangle_{r} \simeq c_{0} \mu_{0} r^{2}+c_{1} \mu_{0}^{2} r^{5}+c_{2} \mu_{0}^{3} r^{8}+\cdots+c_{i} \mu_{0}^{i+1} r^{3 i+2}+\cdots \simeq \mu_{0} r^{2} G_{1}\left(\mu_{0} r^{3}\right)
$$

and, by the same token

$$
\left\langle\mu^{2}\right\rangle_{r}=\sum_{k=2}^{\infty}(-1)^{k} \mu_{0}^{k} \sum_{j=1}^{k-1}\left\langle V_{j} V_{k-j}\right\rangle_{r} \simeq \mu_{0}^{2} r^{4} G_{2}\left(\mu r^{3}\right) .
$$

Similarly one obtains for any $n$

$$
\left\langle\mu^{n}\right\rangle_{r} \simeq \mu_{0}^{n} r^{2 n} G_{n}\left(\mu_{0} r^{3}\right)
$$

so that, requiring the existence of the thermodynamic limit, one obtains as $\mu_{0} \rightarrow 0^{+}$at $r=\infty$

$$
\left\langle\mu^{n}\right\rangle \equiv \lim _{r \rightarrow \infty}\left\langle\mu^{n}\right\rangle_{r}=b_{n} \mu_{0}^{n / 3}
$$

where

$$
b_{n}=\lim _{s \rightarrow \infty} s^{2 n / 3} G_{n}(s) .
$$

We may also reformulate this result as

$$
\left\langle\mu^{n}\right\rangle_{r} \simeq \mu_{0}^{n / 3} F_{n}\left(\mu_{0} r^{3}\right)
$$

where $F_{n}(s)=s^{2 n / 3} G_{n}(s)$ has a finite nonzero limit as $s \rightarrow \infty$. This general form of the moments of $P_{r}\left(\mu ; \mu_{0}\right)$ implies the scaling form (21) with $\beta=-1 / 3, \gamma=3$ and

$$
F_{n}(s)=\int \mathrm{d} u u^{n} F(u, s)
$$

for the probability $P_{r}\left(\mu ; \mu_{0}\right)$ itself.

Let us close this section with one more observation concerning the universality of the scaling law (3) or (21) with respect to the coordination distribution defined by the $f_{z}$ numbers or, equivalently, by the probability generating function $g(\lambda)$. A more careful analysis of the 


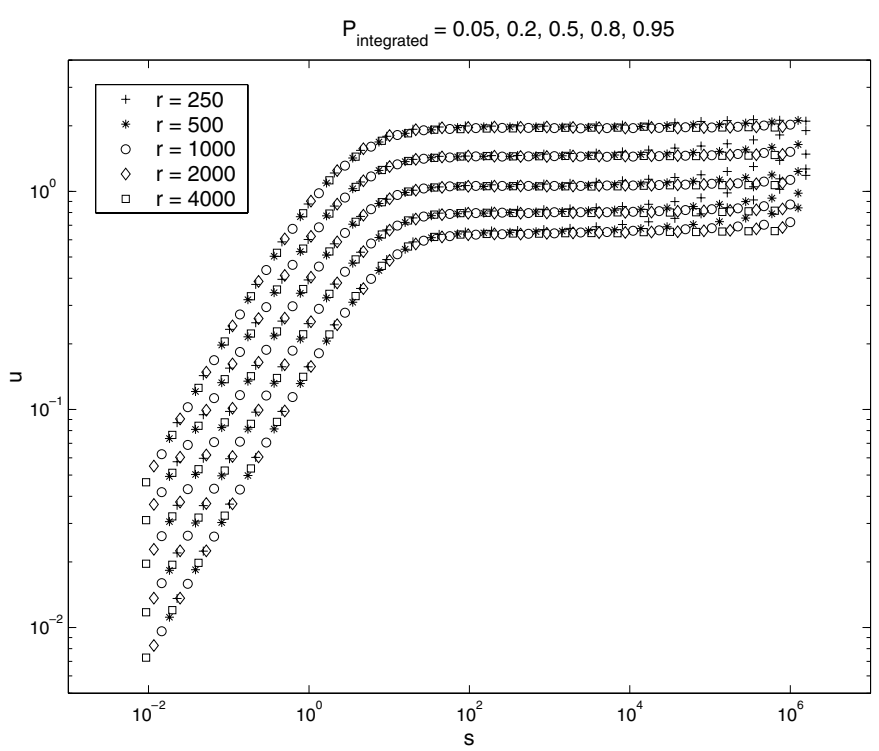

Figure 1. Lines of constant integrated probability from the numerical data.

dominant large $r$ terms in the recursion rules for $V_{n}$ expectations shows that (see the appendix for details)

$$
\left\langle\prod_{j=1}^{k} V_{n_{j}}\right\rangle_{r} \sim \alpha^{\sum_{j} n_{j}} r^{3 \sum_{j} n_{j}-k}
$$

where $\alpha=g^{\prime \prime}(1)$ measures the fluctuations of the coordinations since

$$
\alpha=\sum_{z}(z-2)^{2} f_{z}
$$

We may therefore refine the scaling law (21) to

$$
P_{r}\left(\mu ; \mu_{0}\right) \simeq\left(\alpha \mu_{0}\right)^{-1 / 3} F\left(\left(\alpha \mu_{0}\right)^{-1 / 3} \mu, \alpha \mu_{0} r^{3}\right)
$$

where $F(u, s)$ is a universal scaling function.

\section{Numerical checks}

As an extra check on our derivation and to determine the profile of the universal function $F(u, s)$, we numerically evaluated $P_{r}\left(\mu ; \mu_{0}\right)$ by directly calculating the effective mass $\mu$ for each member of a large set of trees produced by our branching algorithm. For each one of the selected values of $\mu_{0}$ and $r$ we obtained several thousand values of $\mu$ that may be arranged into a histogram providing a discretized estimate of $P_{r}\left(\mu ; \mu_{0}\right) \mathrm{d} \mu$. This procedure does not require much computer time or memory thanks to the recursive structure of the mass composition formula (16). Our results for the integrated probability at the five values $0.05,0.2,0.5,0.8$ and 0.95 are plotted in figure 1 using the scaling variables $u=\mu_{0}^{-1 / 3} \mu$ and $s=\mu_{0} r^{3}$ (with our specific choice of $f_{z}$ we have $\alpha=1$ ). The data show a very good scaling profile with a very well-defined crossover, as $s$ gets larger, from the behaviour typical of small trees, $\mu \propto \mu_{0}$, to that characteristic of large trees, that is $\mu \propto \mu_{0}^{1 / 3}$. The vertical section of the data at 


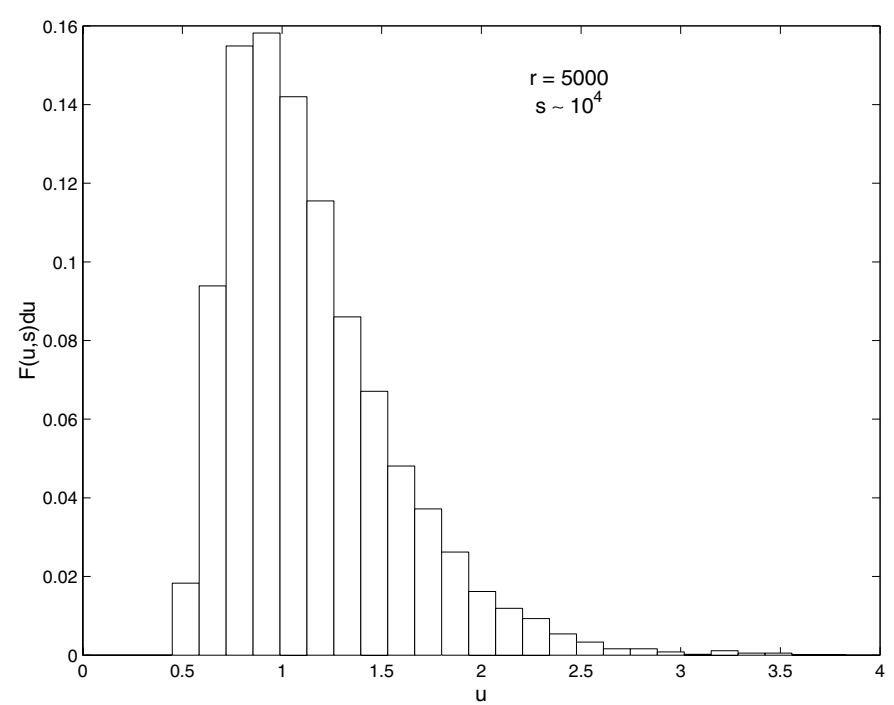

Figure 2. Numerical distribution for $F(u, s)$ for 10000 random trees.

$s \simeq 10^{4}$ provides a good estimate of the probability distribution $F(u, \infty) \mathrm{d} u$ and is reported in figure 2 .

\section{Conclusions and outlook}

Let us remark once more on the two distinct levels of our approach: our proof by induction of equation (33) provides a rigorous basis for the scaling form (21) of $P_{r}\left(\mu ; \mu_{0}\right)$ with $\beta=-1 / 3$ and $\gamma=3$. Thanks to the auto-averaging property, this implies in turn that indeed

$$
\operatorname{Sing} \overline{C\left(\mu_{0}\right)} \propto \mu_{0}^{\bar{d} / 2-1} \quad \bar{d}=4 / 3 .
$$

Moreover, due to the statistical homogeneity of our random trees, we see that the local and average spectral dimensions coincide. On the other hand, if we assume scaling, as natural and common in this context, then the determination $\bar{d}=4 / 3$ follows solely and very simply from the large $r$ asymptotic behaviour of $\left\langle V_{2}\right\rangle_{r}$, that is the average of the sum of squared volumes of all subtrees of the tree. Since the volume $V_{1}$ scales as $r^{\bar{d}_{\mathrm{c}}}$, with $\bar{d}_{\mathrm{c}}=2$, then $V_{2}$ must scale, almost by definition, as $r^{2 \bar{d}_{\mathrm{c}}+1}$; then

$$
\langle\mu\rangle_{r} \simeq \mu_{0} r^{\bar{d}_{\mathrm{c}}}\left(c_{0}+c_{1} \mu_{0} r^{\bar{d}_{\mathrm{c}}+1}+\cdots\right)
$$

which is consistent with scaling only if

$$
\bar{d}=\frac{2 \bar{d}_{\mathrm{c}}}{\bar{d}_{\mathrm{c}}+1} .
$$

Note that this holds true even if $\bar{d}_{\mathrm{c}}<2$, as could happen for different types of bounded trees. We conjectured in [2] that $\bar{d}_{\mathrm{c}} \leqslant 2$ for any bounded tree, with saturation only for random trees. Then automatically $\bar{d} \leqslant 4 / 3$ for any bounded tree, with saturation only for random trees. The general expression (35) was conjectured years ago from very different considerations [9].

Another relevant issue concerns the spectral weight $\bar{w}$ of infinite random trees. This is the coefficient of the $\mu_{0}^{\bar{d} / 2-1}$ singularity of $\overline{C\left(\mu_{0}\right)}$ as $\mu_{0} \rightarrow 0$, that is

$$
\overline{C\left(\mu_{0}\right)} \sim \bar{w} \mu_{0}^{-1 / 3} \text {. }
$$


It is clear from equation (15) and the auto-averaging property that the spectral weight is related to the scaling function $F(u, s)$ by

$$
\bar{w}=\int \frac{\mathrm{d} u}{2 u} F(u, \infty) .
$$

On the other hand, it is known [12] that on any given infinite graph with local spectral dimension $d<2$, the local spectral weight does not really depend on $x$, that is

$$
C_{x x}\left(\mu_{0}\right) \sim w \mu_{0}^{d / 2-1}
$$

for any node $x$. Moreover, thanks to statistical homogeneity, it is clear that on a given tree $w=(2 u)^{-1}$, with $u$ a specific instance of a random variable distributed according to $F(u, \infty)$. Since $F(u, \infty)$ is not $\delta$-like (see the previous section), we have in general $w \neq \bar{w}$, that is the local and average spectral weights differ in general, unlike the spectral dimension. One might wonder then about the auto-averaging property, since $w$ does not fluctuate over the tree. Of course the resolution of this apparent contradiction is in the order of limits: averaging over the infinite graph and small $\mu_{0}$ asymptotics of $C_{x x}\left(\mu_{0}\right)$ are operations that cannot be commuted in general.

These last remarks suggest a further development in the explicit calculation of the universal limit function $F(u, \infty)$. Another interesting direction of research is in the construction of modified tree-generating algorithms such that scaling is preserved with $\bar{d}_{\mathrm{c}}<2$, in order to check the general formula (35).

\section{Acknowledgments}

We thank R Burioni and D Cassi for many valuable comments and suggestions.

\section{Appendix. Asymptotic behaviour of moments}

First of all let us write the explicit form of $\mathcal{F}_{n}$

$$
\mathcal{F}_{n}\left(V_{1}, V_{2}, \ldots, V_{n}\right)=V_{n}+\sum_{k=2}^{n} \sum_{n_{1}=1}^{n-1} \cdots \sum_{n_{k}=1}^{n-1} \delta\left(n-\sum_{j=1}^{k} n_{i}\right) \prod_{j=1}^{k} V_{n_{j}} .
$$

In the case without the non-extinction condition we will prove that

$$
\left\langle\prod_{j=1}^{k} V_{n_{j}}\right\rangle_{r}^{\circ} \sim \alpha^{\sum_{j} n_{j}-1} r^{3 \sum_{j} n_{j}-k-1} .
$$

First of all let us prove this equation when all $n_{j}=1$; the case $k=1$ is known from equation (28) while for arbitrary $k$ is proved by induction; in fact if

$$
\left\langle V_{1}^{j}\right\rangle_{r}^{\circ} \sim \alpha^{j-1} r^{2 j-1}
$$

is assumed for $j \leqslant k$ we have

$$
\begin{aligned}
\left\langle V_{1}^{k+1}\right\rangle_{r+1}^{\circ}= & \sum_{z} f_{z} \sum_{V_{1,1}, V_{1,2}, \ldots, V_{1, z-1}}\left(1+\sum_{i=1}^{z-1} V_{1, i}\right)^{k+1} \prod_{i=1}^{z-1} P_{r}^{\circ}\left(V_{1, i}\right) \\
& =\sum_{z} f_{z} \sum_{V_{1,1}, V_{1,2}, \ldots, V_{1, z-1}}\left(\left(\sum V_{1, i}\right)^{k+1}+k\left(\sum V_{1, i}\right)^{k}+\cdots+1\right) \prod_{i=1}^{z-1} P_{r}^{\circ}\left(V_{1, i}\right)
\end{aligned}
$$




$$
\begin{aligned}
= & \left\langle V_{1}^{k+1}\right\rangle_{r}^{\circ}+\alpha \sum_{j=1}^{k}\left\langle V_{1}^{j}\right\rangle_{r}^{\circ}\left\langle V_{1}^{k+1-j}\right\rangle_{r}^{\circ} \\
& +g^{\prime \prime \prime}(1) \sum_{j_{1}, j_{2}, j_{3}=1}^{k}\left\langle V_{1}^{j_{1}}\right\rangle_{r}^{\circ}\left\langle V_{1}^{j_{2}}\right\rangle_{r}^{\circ}\left\langle V_{1}^{j_{3}}\right\rangle_{r}^{\circ} \delta\left(k+1-j_{1}-j_{2}-j_{3}\right)+\cdots \\
& +k\left\langle V_{1}^{k}\right\rangle_{r}^{\circ}+\alpha \sum_{j=1}^{k}\left\langle V_{1}^{j}\right\rangle_{r}^{\circ}\left\langle V_{1}^{k-j}\right\rangle_{r}^{\circ}+\text { lower order terms } \\
\sim & \left\langle V_{1}^{k+1}\right\rangle_{r}^{\circ}+\alpha^{k} r^{2 k}
\end{aligned}
$$

where all addends which consist of a product of three or more averages and those that come from lower powers of $\left(\sum V_{1, i}\right)$ are negligible in the limit $r \rightarrow \infty$ thanks to equation (A2), so that

$$
\left\langle V_{1}^{k+1}\right\rangle_{r}^{\circ} \sim \alpha^{(k+1)-1} r^{2(k+1)-1}
$$

extending equation (A2) from $k$ to $k+1$.

Now, let us prove equation (A1) by induction; in this case the induction is on the quantity $N=3 \sum_{j=1}^{k} n_{j}-k$. Let us denote by $\bar{n}$ the greatest among $n_{1}, n_{2}, \ldots, n_{k}$; we have

$$
\begin{aligned}
\left\langle\prod_{j=1}^{k} V_{n_{j}}\right\rangle_{r+1}^{\circ}= & \sum_{z} f_{z} \sum_{\begin{array}{c}
V_{1,1}, \ldots, V_{1, z-1} \\
V_{2,1}, \ldots, V_{2, z-1} \\
V_{\bar{n}, 1}, \ldots, V_{\bar{n}, z-1}
\end{array}} \prod_{j=1}^{k}\left(\delta_{n_{j}, 1}+\sum_{i=1}^{z-1} \mathcal{F}_{n_{j}}\left(V_{1, i}, \ldots, V_{n_{j}, i}\right)\right) \prod_{i=1}^{z-1} P_{r}^{\circ}\left(V_{1, i}, \ldots, V_{\bar{n}, i}\right) \\
= & \left\langle\prod_{j=1}^{k} V_{n_{j}}\right\rangle_{r}^{\circ}+\sum_{j=1}^{k} \sum_{l=1}^{n_{j}-1}\left\langle V_{l} V_{n_{j}-l} \prod_{j^{\prime} \neq j} V_{n_{j^{\prime}}}\right\rangle_{r}^{\circ} \\
& +\alpha \sum_{S}\left\langle\prod_{j \in S} V_{n_{j}}\right\rangle_{r}^{\circ}\left\langle\prod_{j \notin S} V_{n_{j}}\right\rangle_{r}^{\circ}+\text { lower order terms } \\
\sim & \left\langle\prod_{j=1}^{k} V_{n_{j}}\right\rangle_{r}^{\circ}+\alpha^{\sum_{j} n_{j}-1} r^{3 \sum_{j} n_{j}-k-2}
\end{aligned}
$$

where $S$ are all the possible ordered subsets of $\left\{n_{1}, n_{2}, \ldots, n_{k}\right\}$. Now equation (A1) follows but a few remarks are needed. First, all terms on the right-hand side involve averages whose value of $N$ is smaller than those on the left-hand side. Then all the terms that are not explicitly written are proportional to lower powers of $r$ : some of them in fact consist of the product of three or more averages (each of them carries a ' -1 ' in the exponent), some others are expressions with more than $k+1$ factors $V_{n}$, and finally some others come from products involving the $\delta_{n_{j}, 1}$.

We turn now to the probability $P^{\bullet}\left(V_{1}, \ldots, V_{n}\right)$ with non-extinction condition for which the recursion reads

$$
\begin{aligned}
P_{r+1}^{\bullet}\left(V_{1}, \ldots, V_{n}\right) & =\sum_{z} \tilde{f}_{z} \sum_{\substack{V_{1,1}, \ldots, V_{1, z-1} \\
V_{2,1} \ldots, V_{2, z-1} \\
V_{n, 1} \ldots, V_{n, z-1}}} P_{r}^{\bullet}\left(V_{1,1}, \ldots, V_{n, 1}\right) \prod_{i=2}^{z-1} P_{r}^{\circ}\left(V_{1, i}, \ldots, V_{n, i}\right) \\
& \times \prod_{k=1}^{n} \delta\left(V_{k}-\delta_{n, 1}-\sum_{j=1}^{z-1} \mathcal{F}_{k}\left(V_{1, j}, \ldots, V_{k, j}\right)\right)
\end{aligned}
$$


We will prove that

$$
\left\langle\prod_{j=1}^{k} V_{n_{j}}\right\rangle_{r}^{\bullet} \sim \alpha^{\sum_{j} n_{j}} r^{3 \sum_{j} n_{j}-k} .
$$

Some special cases, including $k=n_{1}=1$ have already been shown in section 5 , while the general case is proved by induction. In fact the recurrence for $\left\langle\prod_{j=1}^{k} V_{n_{j}}\right\rangle_{r}^{\bullet}$ reads (writing only leading order terms, as before)

$$
\begin{aligned}
\left\langle\prod_{j=1}^{k} V_{n_{j}}\right\rangle_{r+1}^{\bullet}= & \sum_{z}(z-1) f_{z} \sum_{\substack{V_{1,1}, \ldots, V_{1, z-1} \\
V_{2,1}, \ldots, V_{2, z-1} \\
V_{\bar{n}, 1}, \ldots, V_{\bar{n}, z-1}}}^{k}\left(\delta_{n_{j}, 1}+\sum_{i=1}^{z-1} \mathcal{F}_{n_{j}}\left(V_{1, i}, \ldots, V_{n_{j}, i}\right)\right) \\
& \times P_{r}^{\bullet}\left(V_{1,1}, \ldots, V_{\bar{n}, 1}\right) \prod_{i=2}^{z-1} P_{r}^{\circ}\left(V_{1, i}, \ldots, V_{\bar{n}, i}\right) \\
= & \left\langle\prod_{j=1}^{k} V_{n_{j}}\right\rangle_{r}^{\bullet}+\sum_{j=1}^{k} \sum_{l=1}^{n_{j}-1}\left\langle V_{l} V_{n_{j}-l} \prod_{j^{\prime} \neq j} V_{n_{j^{\prime}}}\right\rangle_{r}+\alpha\left\langle\prod_{j=1}^{k} V_{n_{j}}\right\rangle_{r}^{\circ} \\
& +\alpha \sum_{S}\left\langle\prod_{j \in S} V_{n_{j}}\right\rangle_{r}\left\langle\prod_{j \notin S} V_{n_{j}}\right\rangle_{r}^{+}+\text {lower order terms } \\
\sim & \left\langle\prod_{j=1}^{\bullet} V_{n_{j}}\right\rangle_{r}+\alpha^{\sum_{j} n_{j}} r^{3 \sum_{j} n_{j}-k-1}
\end{aligned}
$$

with the same remarks as in the previous case.

Finally the recursion for the probability $P\left(V_{1}, \ldots, V_{n}\right)$ reads

$$
\begin{aligned}
P_{r+1}\left(V_{1}, \ldots, V_{n}\right) & =\sum_{z} f_{z} \sum_{\substack{V_{1,1}, \ldots, V_{1, z} \\
V_{2,1}, \ldots, V_{2, z} \\
V_{n, 1}, \ldots, V_{n, z}}} P_{r}^{\bullet}\left(V_{1,1}, \ldots, V_{n, 1}\right) \prod_{i=2}^{z} P_{r}^{\circ}\left(V_{1, i}, \ldots, V_{n, i}\right) \\
& \times \prod_{k=1}^{n} \delta\left(V_{k}-\delta_{n, 1}-\sum_{j=1}^{z} \mathcal{F}_{k}\left(V_{1, j}, \ldots, V_{k, j}\right)\right)
\end{aligned}
$$

and, following the same steps as before,

$$
\begin{aligned}
&\left\langle\prod_{j=1}^{k} V_{n_{j}}\right\rangle_{r+1}= \sum_{z} f_{z} \sum_{\substack{V_{1,1}, \ldots, V_{1, z} \\
V_{2,1}, \ldots, V_{2, z} \\
V_{\bar{n}, 1}, \ldots, V_{\bar{n}, z}}} \prod_{j=1}^{k}\left(\delta_{n_{j}, 1}+\sum_{i=1}^{z} \mathcal{F}_{n_{j}}\left(V_{1, i}, \ldots, V_{n_{j}, i}\right)\right) \\
& \times P_{r}^{\bullet}\left(V_{1,1}, \ldots, V_{\bar{n}, 1}\right) \prod_{i=2}^{z} P_{r}^{\circ}\left(V_{1, i}, \ldots, V_{\bar{n}, i}\right) \\
&=\left\langle\left.\prod_{j=1}^{k} V_{n_{j}}\right|_{r} ^{\bullet}+\right.\text { lower order terms } \\
& \sim \alpha^{\sum_{j} n_{j}} r^{3 \sum_{j} n_{j}-k} .
\end{aligned}
$$




\section{References}

[1] Alexander S and Orbach R 1982 J. Phys. Lett. 43625

[2] Destri C and Donetti L 2002 J. Phys. A: Math. Gen. 355147

[3] Burioni R and Cassi D 1996 Phys. Rev. Lett. 761091

[4] Cassi D 1996 Phys. Rev. Lett. 762941

Burioni R, Cassi D and Vezzani A 1999 Phys. Rev. E 601500

[5] Essam J W 1980 Rep. Prog. Phys. 43833

[6] Jonsson T and Wheater J F 1998 Nucl. Phys. B 515549

[7] Leyvraz F and Stanley E 1983 Phys. Rev. Lett. 512048

[8] Kesten H 1987 Ann. Inst. H Poincaré B 22425

[9] Havlin S and Ben-Avraham D 1987 Adv. Phys. 36 695-798

[10] Burioni R and Cassi D 1995 Phys. Rev. E 512865

[11] Donetti L 2002 Preprint cond-mat/0206149

[12] Hattori K, Hattori T and Watanabe H 1987 Prog. Theor. Phys. Suppl. 92108

[13] Burioni R, Cassi D and Vezzani A 1999 J. Phys. A: Math. Gen. 325539 